\title{
DYNAMIC SEMANTIC ONTOLOGY GENERATION: A PROPOSAL FOR SOCIAL ROBOTS
}

\author{
Javier Sevilla-Salcedo, M. A. Quispe-Flores, Sara Carrasco-Martínez, Jaime Gómez-Jiménez \\ J. C. Castillo, Á. Castro-González, M. Malfaz, Miguel A. Salichs \\ RoboticsLab, Departamento de Ingeniería de Sistemas y Automática, \\ Universidad Carlos III de Madrid \\ \{jasevill, mquispe, sacarras\}@pa.uc3m.es, 100383149@alumnos.uc3m.es \\ \{jocastil, acgonzal, mmalfaz, salichs\}@ing.uc3m.es
}

\begin{abstract}
During a human-robot interaction by dialogue/voice, the robot cannot extract semantic meaning from the words used, limiting the intervention itself. Semantic knowledge could be a solution by structuring information according to its meaning and its semantic associations. Applied to social robotics, it could lead to a natural and fluid human-robot interaction. Ontologies are useful representations of semantic knowledge, as they capture the relationships between objects and entities. This paper presents new ideas for ontology generation using already generated ontologies as feedback in an iterative way to do it dynamically. This paper also collects and describes the concepts applied in the proposed methodology and discusses the challenges to be overcome.
\end{abstract}

Keywords: Ontology Generation, Semantic Knowledge, Hierarchical Clustering, Social Robots, Decision-Making System, Human-Robot Interaction, Cognitive Stimulation

\section{INTRODUCTION}

Throughout the paper, semantic knowledge is defined as a domain that includes everything known about a subject or object, including language semantics (the meaning of words, objects and entities) [10]. In recent years there has been a trend towards integrating high-level information in various computer applications, which, in turn, improves the adaptability to more real-life situations.

In semantic knowledge, one of the biggest problems over the years has been the correct approach to its representation because of the abstract nature of concepts [15]. In this context is where ontologies come in. Ontologies are representation tools for defining and describing objects, properties and relationships in a knowledge domain. Once solved the problem of representation, a new paradigm emerges in the generation of a semantic database that brings together these concepts.

Many fields of knowledge have included ontologies in their development, such as the IoT, where we find the ROCAS project (Reasoning in the Cloud Applying Semantics), which aims to provide a distributed semantic reasoning system based on cloud computing [6]. RoboEarth is another example, this time focused on robotics, of the use of semantic knowledge. RoboEarth is a worldwide web for robots: a giant network and database repository where robots can share information and learn from each other [29]. Another application is semantic navigation, where a mobile robot can move around a house, taking into account the use of each room. In a practical case, given the command "I would like to have a cold drink", the robot would move to the kitchen by reasoning that the cold drink is in the fridge, which is in the kitchen [5].

One of the problems encountered in this specific application is the user's manual input of the ontology, which implies a meticulous and tedious procedure of defining a specific ontology for each environment or topic [4]. In this context, a further stage is to establish a methodology for gathering this knowledge and adapting it to the representation system, such as ontologies.

Therefore, the main goal of this paper is to present our first ideas of a new ontology generation model inspired by the breakthroughs in machine learning to make the process more dynamic and applicable on a larger scale for generating semantic knowledge.

The rest of the paper is structured as follows: A brief overview of the main key concepts underlying the methodology is presented in section 2 , followed by our approach and the challenges to be faced in section 3. Section 4 includes a discussion about possible applications in social robotics. Finally, section 5 comments on the conclusions drawn from this article.

\section{STATE OF THE ART}

This section gathers previous knowledge related to the generation of ontologies. Therefore, predefined concepts and methodologies that facilitate the procedure and understanding of semantic 


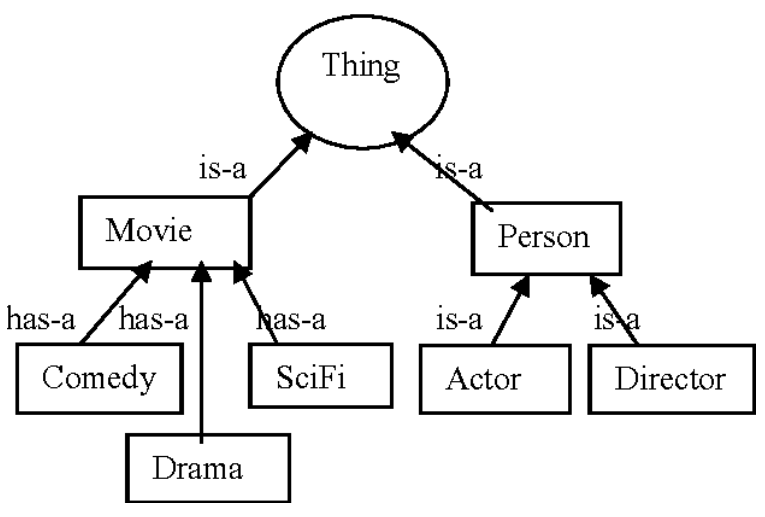

Figure 1: Simple movie ontology [22]

knowledge generation are discussed in this section.

The first matter to deal with is the representation of semantic knowledge. In this sense, ontology is one of the best tools to obtain representations of knowledge through hierarchies of concepts [8]. In many fields, they are used to frame theories, research and implementations.

In addition, to generate ontologies, we will need a tool that, from diverse literature, can classify and group the existing terms, objects and relations and thus create the ontology from it. Clustering has this purpose; this technique generates groupings (clusters) from unstructured data.

However, before clustering, it is advisable to preprocess the text to remove words that lack semantic information, such as articles. It is also helpful to make a primary distinction between verbs, adjectives, nouns or adverbs. Natural Language Processing Tools allow the user to perform these and other functionalities.

\section{$2.1 \quad$ ONTOLOGIES}

In knowledge engineering, ontologies collect and define the relations, rules and hierarchies in our language and the conceptualisation of an environment [7]. Its main characteristic is the establishment of properties and relationships between entities or concepts [30]. Its use limits complexity and organises information in a solution-oriented way [9]. Figure 1 shows an simple example of movie ontology. The figure depicts the relationships between the different classifications and attributes.

Due to the enhanced performance obtained using ontologies reported on different applications discussed below, its use becomes essential since it deals with the uncertainty and heterogeneity of unstructured data. Numerous developments and applications focus on the treatment and management of ontologies. For example, semantic integration has been studied, looking at the possible combination between ontologies and the representation of concept maps based on ontologies [19].

\subsection{CLUSTERING}

Once the system of semantic knowledge representation well defined and established, the next step is to explore the possible methodology to perform this hierarchical categorisation of conceptualisations in an automated manner to produce ontologies.

For this purpose, clustering appears, a technique commonly used in the analysis of statistical data and, in this case, widely used in the field of machine learning and data mining. Clustering is typically an unsupervised learning method whose main objective is defining and establishing new categories or groups (clusters) in a dataset based on recognising patterns. This concept goes hand in hand with ontologies, as Tyron and Bailey stated in 1970, understanding our world requires conceptualising the similarities and differences between the entities that compose it [1][24].

Within clustering, we can find different approaches and methodologies. Going into its taxonomy, included in Figure 2, the first distinction occurs between hierarchical and partitional algorithms. The first algorithms define nested clusters and subclusters within the sets at each level, typically represented by a dendrogram, a graphical representation in the form of a tree that organises data into subcategories. Partitionals, on the other hand, obtain a classification or grouping at a single hierarchy level rather than a clustering structure, which sometimes has drawbacks such as the number of clusters. Though dealing with vast amounts of data is computationally more feasible than acquiring a hierarchical structure [14].

\subsection{ONTOLOGY-BASED CLUSTERING}

In 2001, Maedche and Staab presented an ontology learning framework using different semiautomatic ontology building tools [17]. The framework includes several practical applications such as structure combination, model extraction, pruning and refinement. In addition, a graphical user interface collects all the development while allowed the visualisation of the generated structures and the manual modification at any level if necessary. A year later, together with A. Hotho, they redefined the model extraction step and focused on ontology-based document clustering, introducing a new development in text pre-processing be- 


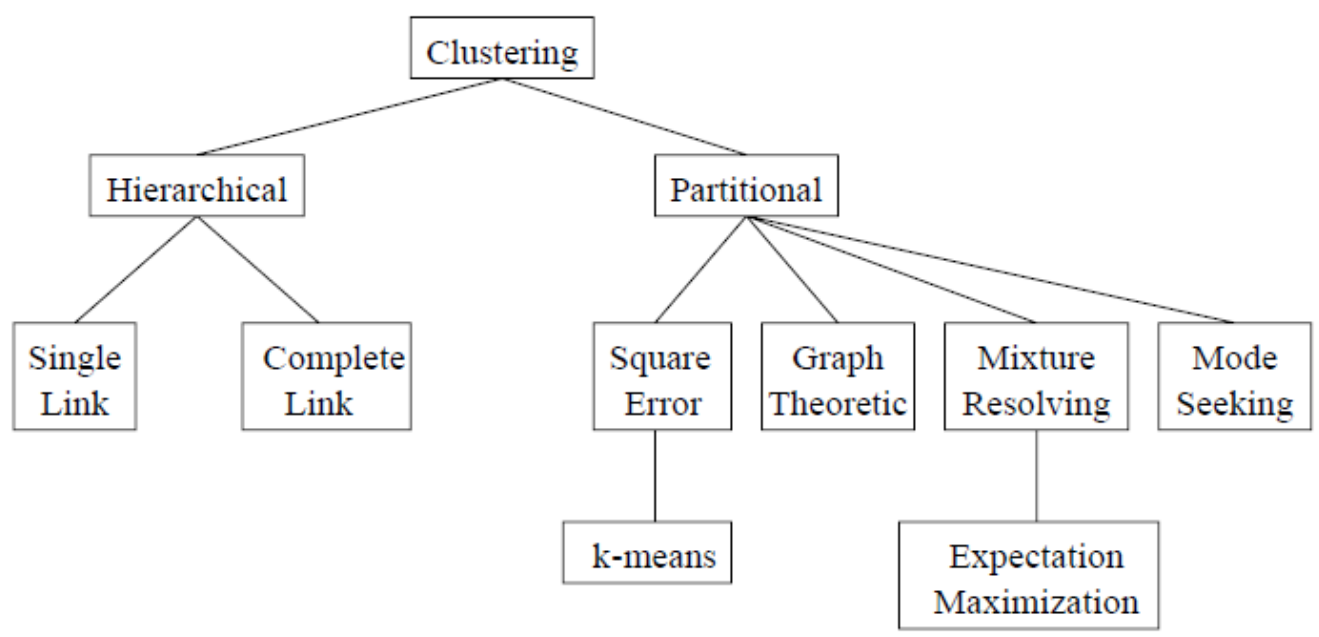

Figure 2: A taxonomy of clustering approaches [14]

fore the primary analysis [12]. The Concept Selection and Aggregation (COSA) strategy consisted of pre-mapping concepts using a natural language processing system before heterarchy usage to provide suitable aggregations for further clustering [13].

Breaux et al. proposed in 2005 a different approach to the problem based on the developments and advances made by Hotho et al. [13], in which they demonstrated the positive impact of the use of ontologies for text filtering. In this case, they used VIPAR, an automatic hierarchical clustering system previously tested for the use of texts to obtain dendrograms [21]. The developed system used the ontology during the filtering phase of the acquisition process in order to reduce and adjust in a controlled way the matching relations between the ontology employed and the documents [2].

In order to systematically identify clusters of developmental disorders in children and to be able to represent them through ontologies, Peleg et al. proposed the Onto-clust methodology in 2009 [20]. This approach focused on combining clustering with ontological methods, using the ontology as a refinement or adjustment of the clustering results by adding labels and improving the classification found in the clusters obtained. It is a matter of providing human feedback (ontological evidence) to the output, making it a semi-supervised process where the desired large-scale classification results are known in prior knowledge.

In 2013, Ravishankar et al. worked on a new approach to extracting information from text. The development uses two different methods to reshape the information in such a way as to facilitate the retrieval of information. First of all, it pre-processes the text, then a first categorisa- tion based on ontology-based decision trees is performed so that the document gets represented as a hierarchy of terms and concepts. Once obtained the representation, goes through a second clustering process, based on the k-means algorithm, which, being fed with a previous hierarchy, offers better classification results compared to a process without ontologies [23].

\subsection{NATURAL LANGUAGE PROCESSING TOOLS}

In the Natural Language Processing (NLP) field, we find several tools to develop the text preprocessing and make it suitable for further processing. The Natural Language Toolkit (NLTK) library allows the user to process texts, division, conversion, lexical treatment, as well as classification by keywords [18]. Additionally, one of the databases on which this library relies is WordNet, an English lexical database created by George Armitage Miller, which groups English words into sets of synsets, a set of synonyms that share a common meaning. The most significant property of WordNet is the storage of semantic relations between synsets [31].

Within the development of tools focused on NLP and treatment appears the proposal adopted by Google with its Cloud Natural Language, a product that allows the extraction of semantic text information without initial structuring based on its machine learning models. The firm offers in its catalogue both a Natural Language API, which provides previously trained models that allow the analysis and classification of entities or syntactic analysis and the AutoML Natural Language technology, which provides the training of its personalised models, characterising and optimising the 
results according to the user's needs [3].

\section{OUR APPROACH}

The approach proposed in this work aims to generate ontologies dynamically with the primary goal of creating models about general knowledge o be applied later to a social robot. We intend to develop a framework based on semantic knowledge capable of generating its representation from numerous texts. Its implementation implies selfgrowth, as each result feeds the following iterations, thereby extending its scope.

Inspired by the meta-learning philosophy and taking advantage of current breakthroughs in artificial intelligence, the purpose is that the proposed approach is capable of learning to learn knowledge and hence become flexible when it comes to improving and optimising its performance iteratively [11].

Our proposal presents a semi-supervised automatic model that updates its input by filtering it with the ontologies previously generated in the preprocessing stage, based on the developments made by Hotho et al. [13]. In this way, the previously generated categories are searched, thus alleviating the processing and generation of new categorisations. The initial idea is to focus on texts from a previously known and mastered field of knowledge to check the relevance and performance of the text. The ontological filtering of the first iterations will be almost non-existent as it does not feed on ontologies manually. However, with the passage of several iterations and document classifications, the filtering will gain weight and relevance, thus optimising its performance and the ontology itself that feeds the preprocessing phase.

Figure 3 includes a block diagram describing the different steps followed by the model. The input to the system is the text documents from which the knowledge gets extracted. The first block represents the NLP pre-processing, where the text is prepared by synthesising the content and analysing it word by word. Subsequently, we find the ontological filtering, which filters and prepares hierarchically the rest of the information found in the text. Finally, we have the ontology-based clustering, which generates the relevant classifications resulting in the final ontology, which will feed the filtering in the following iterations.

\subsection{CHALLENGES}

In order to achieve our goal, there are several challenges we have to face. Starting with ontologies,

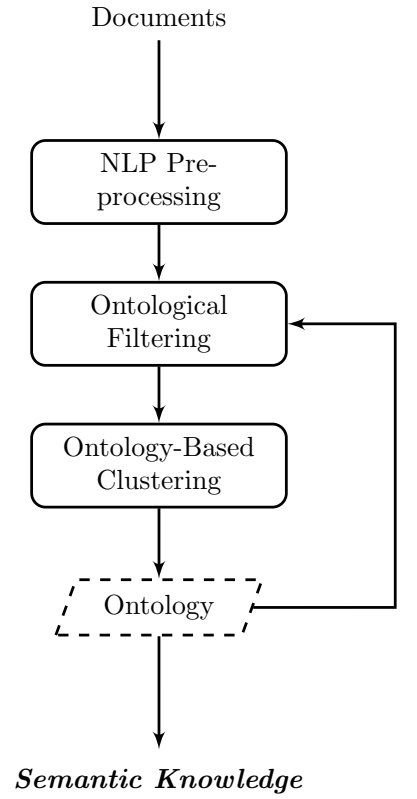

Figure 3: Dynamic Semantic Ontology Generation block diagram

today, there are many languages for their construction. Due to future application, this coding must be suitable for easy transferability. The ideal solution would be to stick to an already defined and widely used language, thus ensuring its robustness and facilitating the possible scalability of ontologies to other general-purpose applications.

Within NLP, we have to take into account certain adverse factors. We need a processing method that we can easily integrate into our system. Furthermore, we need to ensure optimal and robust processing of the texts. To this end, the use of the NLP tools discussed above is under consideration. Additionally, we should consider the language since most of the NLP tools get developed only in English.

To properly design the clustering phase of our system, we need a model that fits our needs. In order to build the ontologies, we need clustering to obtain relevant results, including the hierarchical relationships between the elements found. In this way, it will be possible to build an ontology faithful to the semantics.

One of the main issues encountered in applications created from a meta-learning approach is generalisation. Since the system is building its knowledge from the ground up and feeding it back each iteration, there may come a time when the system is compromised, and a self-suggestion appears that hinders the ability to build and identify new categorisations in its semantic structure.

This problem is nothing new for machine learning 
classification applications, where once the model has a certain number of classes defined, it will value and weight the percentages of belonging to each of the classes but does not consider that one sample might not belong to any predefined class. One approach nowadays is Active Learning, where the algorithm can choose the training data from which it learns. The model can interact with the user to introduce new labels to unidentified outputs [26][27].

Within generalisation, the model training data is crucial for its task. Depending on the topic to be categorised, the texts must be strictly related to that specific topic to extract the appropriate semantic structures. However, there risk within carefully choosing the texts to be fed to the model, as it might overfit. The correct approach is to use texts related to the topic but not previously reviewed to obtain a categorisation naturally and hierarchically. Besides, throughout the learning process, the model needs some randomness.

This randomisation benefits the system in selecting the texts to feed the system and possibly in the previously mentioned ontological filtering phase. Such filtering should contain a weighting that allows generalising the relations found in its structures while not negatively biasing the preprocessing with the ontologies.

Moving on to the challenges to be faced, one of the main challenges from an engineering point of view is the high computational cost of training a system that increases its size with each iteration due to the integration of the feedback of new ontologies for filtering, which will exponentially increase the expenditure of resources. Fortunately, high-performance GPUs are now publicly available thanks to technological advances that can significantly alleviate the computational cost.

\section{APPLICATION TO SOCIAL ROBOTICS}

Combining low-level data with high-level knowledge in a framework would optimise the robot's decision-making process [16]. In robotic navigation, semantic navigation has been studied for some time with promising results. A mobile robot with integrated semantic knowledge offers the user a higher level of understanding and reasoning without working with position targets typically used in the past. Instead, it is possible to express the request for an object or action, at which moment the system will associate that request with the location associated with it through the semantics [5]. For example, as mentioned in the introduction, we could ask the robot for "something
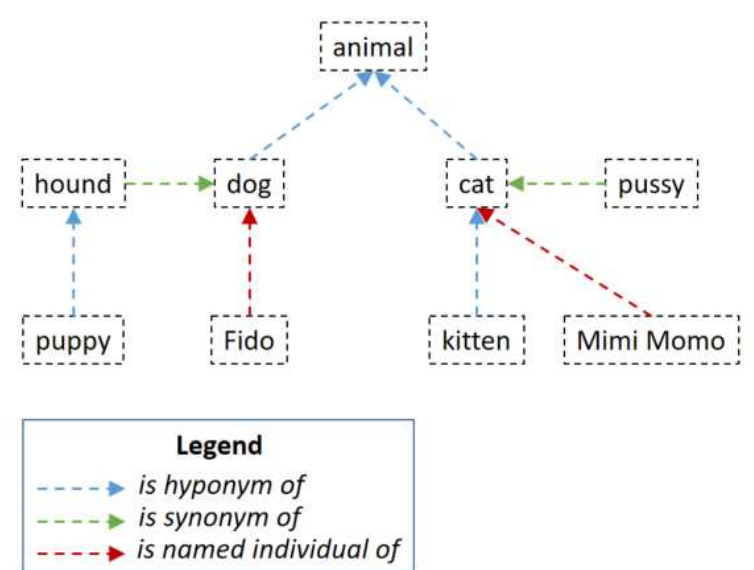

Figure 4: An animal ontology example [28]

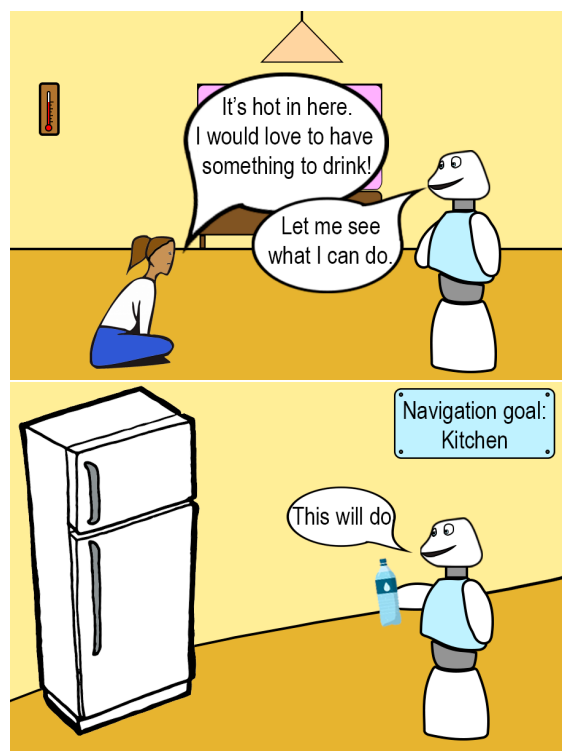

Figure 5: Semantic Navigation example

cold to drink", and the mobile robot, through semantics, would make the cold drink connection, which is in the fridge, and therefore in the kitchen, as reprensented in figure 5 .

We intend to extrapolate it to social robotics, where HRI is the device's primary goal. Providing a new layer of reasoning through semantics would allow the system to manage high-level information more efficiently and flexibly. It is worth noting the significance of the social robot being conversational since the potential of using semantic knowledge lies in the communicative interaction with the user. For example, in a conversation with a user, the robot could use different synonyms or even expressions to refer to the same term if the user does not understand it in the first instance. Although this process can be bidirectional, the robot's oral comprehension can also be made more flexible. 


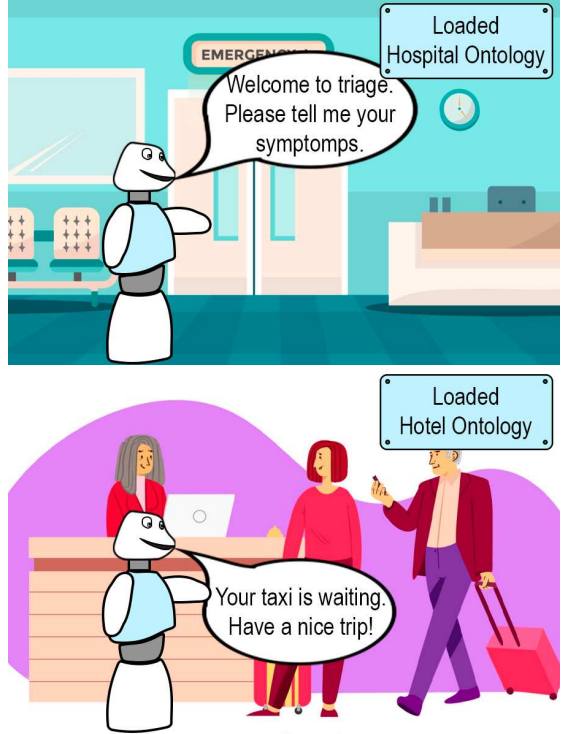

Figure 6: Environment Adaptation example

For example, following the ontology shown in figure 4, the user could use hound, and the robot would still understand that the user is referring to a dog. Using Fido, the robot would understand that the user is referring to a specific $d o g$, an animal.

Using the same ontology example presented in the figure, we can refer to cognitive stimulation with people with neurodegenerative diseases. For example, the patient has to distinguish dogs and cats in a group of animal pictures. We could add new attributes for more intense stimulation to make new classifications such as the type of food (carnivores, herbivores, omnivores) or their natural habitat (seabed, savanna, desert, among others).

Additionally, with semantic knowledge, distinctions could be made in the grammar used depending on the robot's environment. For example, if we were in a hospital, we could load a specific ontology about medicine and assistance to the robot. On the other hand, if we were in a hotel, we could load the ontology specific to the hotel, including information about reservations, rooms or tourist information about the city, as shown in figure 6 .

Based on and inspired by these advantages, we intend to frame this approach in our research group, focusing on the research and development of social robots. The extrapolation and incorporation of semantic knowledge in social robots, such as Mini, shown in Figure 7, can be a substantial step forward in terms of smooth and natural HRI [25].

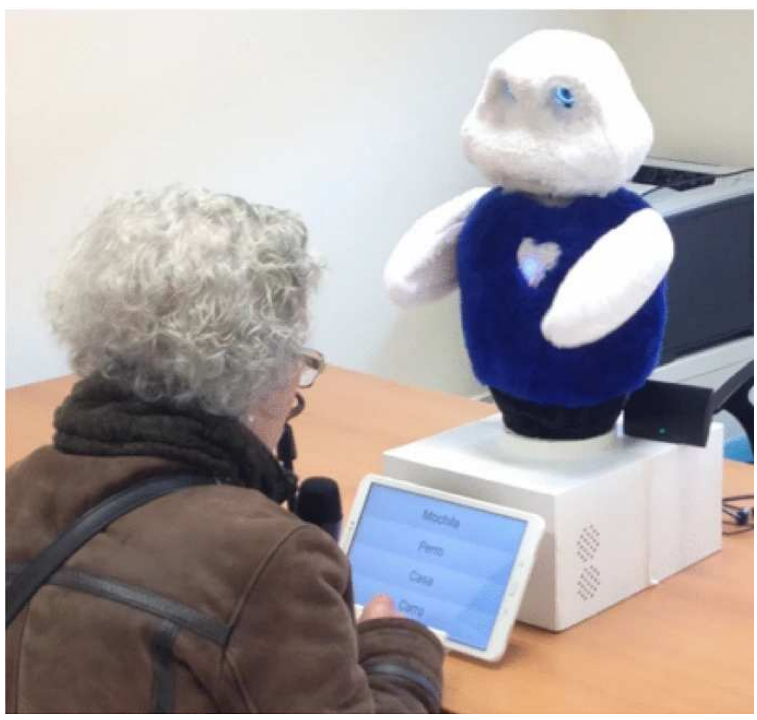

Figure 7: The robot Mini during an interaction with an elderly person [25]

\section{CONCLUSIONS}

Following our line of research, this paper presents the first ideas of a new dynamic approach to semantic knowledge generation through ontology clustering and NLP. With the focus on applying semantic knowledge generated with social robots, as previously mentioned. The proposed methodology offers a semi-supervised model capable of feeding back the previously generated knowledge, thus dynamically building ontologies that can capture the conceptualisation of our environment.

We intend to develop the described model and generate general-purpose ontologies from the literature on different topics in future work. Hence, we may obtain specific ontologies for different topics, applications and environments. Therefore, the robot could selectively use the generated semantic knowledge.

\section{Acknowledgement}

This work was supported by project Robots sociales para estimulación física, cognitiva y afectiva de mayores (ROSES) RTI2018-096338-B-I00 funded by Agencia Estatal de Investigación (AEI), Ministerio de Ciencia, Innovación y Universidades.

\section{References}

[1] K. D. Bailey, "Cluster Analysis," Sociological Methodology, vol. 6, pp. 59-128, 1975. (visited on 06/05/2021).

[2] T. D. Breaux and J. W. Reed, "Using Ontology in Hierarchical Information Clustering," 
en, th Hawaii International Conference on System Sciences, p. 7, 2005.

[3] Cloud Natural Language | Cloud Natural Language, es. [Online]. Available: https:// cloud.google.com/natural - language (visited on 04/26/2021).

[4] J. Crespo, R. Barber, and O. M. Mozos, "Relational Model for Robotic Semantic Navigation in Indoor Environments," en, Journal of Intelligent \& Robotic Systems, vol. 86, no. 3-4, pp. 617-639, Jun. 2017. [Online]. Available: http : //link.springer. com/10.1007/s10846-017-0469-x (visited on $06 / 21 / 2021$ ).

[5] J. Crespo, J. C. Castillo, O. M. Mozos, and R. Barber, "Semantic Information for Robot Navigation: A Survey," en, Applied Sciences, vol. 10, no. 2, p. 497, Jan. 2020, Number: 2 Publisher: Multidisciplinary Digital Publishing Institute. (visited on 02/15/2021).

[6] D. Gayo-Avello, D. Álvarez Gutiérrez, A. Cernuda-del Río, J. Gayo-Avello, L. Vinuesa-Martínez, and N. GarcíaFernández, "The Cooperative Web: A Step towards Web Intelligence," in Web Engineering, G. Goos, J. Hartmanis, J. van Leeuwen, J. M. C. Lovelle, B. M. G. Rodríguez, J. E. L. Gayo, M. del Puerto Paule Ruiz, and L. J. Aguilar, Eds., vol. 2722, Series Title: Lecture Notes in Computer Science, Berlin, Heidelberg: Springer Berlin Heidelberg, 2003, pp. 441444. (visited on 06/21/2021).

[7] T. R. Gruber, "A translation approach to portable ontology specifications," en, Knowledge Acquisition, vol. 5, no. 2, pp. 199-220, Jun. 1993. (visited on 06/02/2021).

[8] — , "Toward principles for the design of ontologies used for knowledge sharing?" en, International Journal of Human-Computer Studies, vol. 43, no. 5, pp. 907-928, Nov. 1995. (visited on $06 / 21 / 2021$ ).
[9] N. Guarino, D. Oberle, and S. Staab, "What Is an Ontology?" en, in Handbook on Ontologies, S. Staab and R. Studer, Eds., Berlin, Heidelberg: Springer Berlin Heidelberg, 2009, pp. 1-17. (visited on 05/27/2021).

[10] P. Gärdenfors, "Semantic Knowledge, Domains of Meaning and Conceptual Spaces," en, in Knowledge and Action, ser. Knowledge and Space, P. Meusburger, B. Werlen, and L. Suarsana, Eds., Cham: Springer International Publishing, 2017, pp. 203-219. (visited on $06 / 17 / 2021$ ).

[11] T. Hospedales, A. Antoniou, P. Micaelli, and A. Storkey, "Meta-Learning in Neural Networks: A Survey," arXiv:2004.05439 [cs, stat], Nov. 2020, arXiv: 2004.05439. (visited on $06 / 05 / 2021)$.

[12] A. Hotho, A. Maedche, and S. Staab, "Ontology-based Text Document Clustering," en, p. 13, 2002.

[13] A. Hotho, S. Staab, and G. Stumme, "Ontologies Improve Text Document Clustering," Oct. 2003.

[14] A. K. Jain, M. N. Murty, and P. J. Flynn, "Data clustering: A review," ACM Computing Surveys, vol. 31, no. 3, pp. 264-323, Sep. 1999. (visited on 06/02/2021).

[15] M. Kaufmann and J. F. Sowa, Principles of Semantic Networks - 1st Edition, 1991. (visited on 06/02/2021).

[16] G. H. Lim, I. H. Suh, and H. Suh, "Ontology-Based Unified Robot Knowledge for Service Robots in Indoor Environments," IEEE Transactions on Systems, Man, and Cybernetics - Part A: Systems and Humans, vol. 41, no. 3, pp. 492-509, May 2011, Conference Name: IEEE Transactions on Systems, Man, and Cybernetics - Part A: Systems and Humans.

[17] A. Maedche and S. Staab, "Ontology learning for the Semantic Web," IEEE Intelligent Systems, vol. 16, no. 2, pp. 72-79, Mar. 
2001, Conference Name: IEEE Intelligent Systems.

[18] Natural Language Toolkit - NLTK 3.6.2 documentation. [Online]. Available: https:// www.nltk.org (visited on 04/26/2021).

[19] N. F. Noy, "Semantic integration: A survey of ontology-based approaches," en, ACM SIGMOD Record, vol. 33, no. 4, pp. 65-70, Dec. 2004. (visited on 05/27/2021).

[20] M. Peleg, N. Asbeh, T. Kuflik, and M. Schertz, "Onto-clust - A methodology for combining clustering analysis and ontological methods for identifying groups of comorbidities for developmental disorders," en, Journal of Biomedical Informatics, vol. 42, no. 1, pp. 165-175, Feb. 2009. (visited on 04/19/2021).

[21] T. E. Potok, "VIPAR: Advanced Information Agents discovering knowledge in an open and changing environment," en, p. 7,

[22] P. Priya and R. Rajalaxmi, "Ontology based semantic query suggestion for movie search," en, undefined, 2013. (visited on 06/21/2021).

[23] N. Ravishankar and S. Raghunathan, Ontology based clustering algorithm for information retrieval. Jul. 2013, Journal Abbreviation: 2013 4th International Conference on Computing, Communications and Networking Technologies, ICCCNT 2013 Pages: 4 Publication Title: 2013 4th International Conference on Computing, Communications and Networking Technologies, ICCCNT 2013.

[24] L. Rokach and O. Maimon, "Clustering Methods," en, in Data Mining and Knowledge Discovery Handbook, O. Maimon and L. Rokach, Eds., Boston, MA: Springer US, 2005, pp. 321-352. (visited on 06/01/2021).

[25] M. A. Salichs, A. Castro, E. Salichs, E. Fernandez, M. Maroto, J. J. Gamboa, S. Marques, J. C. Castillo, F. Alonso, and M. Malfaz, "Mini: A New Social Robot for the El- derly," en, International Journal of Social Robotics, vol. 12, no. 6, pp. 1231-1249, Dec. 2020. (visited on 06/10/2021).

[26] B. Settles, "Active Learning Literature Survey," en, University of Wisconsin-Madison Department of Computer Sciences, Technical Report, 2009, Accepted: 2012-0315T17:23:56Z. (visited on 06/06/2021).

[27] - "Active Learning," Synthesis Lectures on Artificial Intelligence and Machine Learning, vol. 6, no. 1, pp. 1-114, Jun. 2012, Publisher: Morgan \& Claypool Publishers. (visited on $06 / 06 / 2021$ ).

[28] P. Tiwari, ONTOLOGY, en, Dec. 2019. [Online]. Available: https ://medium.com/ Oprince.alobha/ontology-4924049bc21c (visited on 06/22/2021).

[29] M. Waibel, M. Beetz, J. Civera, R. D'Andrea, J. Elfring, D. Gálvez-López, K. Häussermann, R. Janssen, J. Montiel, A. Perzylo, B. Schießle, M. Tenorth, O. Zweigle, and R. V. De Molengraft, "RoboEarth," IEEE Robotics Automation Magazine, vol. 18, no. 2, pp. 69-82, Jun. 2011, Conference Name: IEEE Robotics Automation Magazine.

[30] C. Welty, "Ontology Research," en, AI Magazine, vol. 24, no. 3, pp. 11-11, Sep. 2003, Number: 3. (visited on 06/02/2021).

[31] WordNet | A Lexical Database for English. [Online]. Available: https : //wordnet. princeton.edu (visited on 04/19/2021).

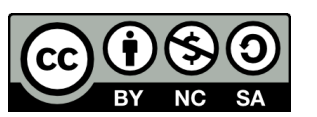
(C) 2021 by the authors. Submitted for possible open access publication under the terms and conditions of the Creative Commons Attribution CC BY-NC-SA 4.0 license (https://creativecommons.org/licenses/by-ncsa/4.0/deed.es). 